\title{
Probabilidad y contratos. Sobre el pragmatismo de Roberto Torretti*
}

\begin{abstract}
Resumen
Pretendo establecer aquí un diálogo con la concepción pragmatista de la probabilidad defendida por Roberto Torretti a partir del enfoque propensionista. En la primera parte del trabajo, quiero mostrar en qué sentido la esperanza matemática formalizaba un principio aristotélico de justicia. En la segunda parte ilustraré, apoyándome en los trabajos de G. Shafer y V. Vovk, cómo iluminar sistemáticamente esa normatividad a partir de una concepción de la probabilidad articulada sobre la teoría de juegos. Veremos así cómo hay una dimensión pragmatista en la probabilidad que cumple con los desiderata de Torretti, aunque no se apoye en simetrías físicas.

Palabras clave: probabilidad, propensiones, contratos, esperanza matemática, Torretti.
\end{abstract}

\section{Probability and Contracts. About Roberto Torretti's Pragmatism}

\begin{abstract}
I want to expand the pragmatist view of probability advocated by Roberto Torretti, drawing on the propensity approach. In the first part, I will show how the concept of mathematical expectation originally formalized an Aristotelian principle of justice. In the second half, I will present the game-theoretic approach to probability developed by Shafer and Vovk, showing how it allows us to interpret the original normativity of mathematical expectations. I will finally discuss how

\footnotetext{
* Recibido: octubre 2016.

** Departamento de Lógica, Historia y Filosofía de la ciencia, Universidad Nacional de Educación a Distancia. Madrid, España. Email: dteira@fsof.uned.es
} 
this latter view contributes to a pragmatist understanding of probability, as Torretti advocates, although with broader foundations than the physical symmetries of standard propensities.

Keywords: probability, propensity, contracts, mathematical expectation, Torretti.

\section{La ejemplaridad de Roberto Torretti ${ }^{1}$}

Me gustaría comenzar el texto explicando el modo en que Roberto Torretti ha sido un ejemplo académico para mí. Aunque sólo sea para comprometerme públicamente a imitarlo -y que puedan avergonzarme si dejo de intentarlo alguna vez. En primer lugar, como ejemplo de filosofar cosmopolita del que tan pocos casos tenemos en el mundo hispano-americano. Cuando llegué a Londres en 2005 a realizar mi (segunda) Tesis doctoral, Torretti era el único filósofo de la ciencia iberoamericano (de su generación y buena parte de las siguientes) que aparecía regularmente en la conversación. En buena parte, por sus muchos años de diálogo internacional con tantos y tantos autores; pero también porque sus ideas reaparecían en el Zeitgeist londinense, a propósito del neopragmatismo de Hasok Chang o el neokantismo de Michela Massimi. Comencé a mantener correspondencia regular con Torretti cuando, a partir de 2009, empecé a editar la revista Theoría, a la que él contribuyó muchas veces como autor y evaluador. Y, para mí, editor novel, fue también ejemplar descubrir la generosidad (iy puntualidad!) de sus colaboraciones, cuando tantos otros simplemente dejaban de contestar mis correos. Finalmente, ya en mi actual destino, la Universidad Nacional de Educación a Distancia, me tocó poner en marcha en 2013 una asignatura de "Historia de las matemáticas" para futuros matemáticos. Buscando manuales, descubrí cómo Torretti había puesto en libre acceso su espléndido El Paraíso de Cantor (Torretti, 1998) y de inmediato se lo propuse a mis estudiantes. Son ya varias las promociones que le agradecen a Torretti no sólo el permitirles usar gratuitamente un texto tan bien editado, sino el modo en que les ha hecho mirar la matemática a través de la Historia y filosofía de la teoría de conjuntos. Apenas pude pasar una tarde con Roberto Torretti y Carla Cordua, en enero de 2013, pero su ejemplaridad académica es una constante inspiración para mí.

Aquella tarde en Santiago me habló de un texto suyo sobre el concepto de probabilidad (Torretti, 2003), en el que dialogaba, entre otros, con Donald

\footnotetext{
${ }^{1}$ Agradezco a Roberto Torretti y Antonio Heras sus comentarios sobre el texto, así como el patrocinio del proyecto de investigación FFI2014-57258-P
} 
Gillies, mi director. La conversación se interrumpió y me gustaría retomarla aquí para prolongar aquel encuentro tan grato. Torretti presentaba en el texto las tres concepciones principales de la probabilidad (frecuentista, subjetivista y propensionista) con su acostumbrada claridad y rigor histórico, discutiendo los principales argumentos a favor y en contra de cada una de ellas. Dos son las tesis de Torretti que sirven de punto de partida a mi análisis. Sostiene, en primer lugar, que "la noción moderna de probabilidad constituye un asunto especialmente atractivo para cualquiera que se interese en la historia y la historicidad de la razón" (Torretti, 2003). Efectivamente, aunque podemos encontrar intuiciones sobre el azar ya desde la antigüedad clásica, es en el siglo XVII cuando comienza a articularse matemáticamente en el concepto moderno de probabilidad. ¿Por qué entonces? ¿Y cómo es que, pese a la claridad axiomática con la que hoy la definimos, se han podido desarrollar tres interpretaciones filosóficamente contrapuestas?

En su discusión, Torretti reivindica su propia perspectiva pragmatista. Contra el frecuentismo, objeta que reducir la probabilidad a frecuencias infinitas de sucesos recurrentes incurre en un dilema moral: "si no tiene sentido hablar de la probabilidad de un suceso singular, ¿qué queda de la probabilidad como guía de la vida?" (Torretti, 2003: 13). Contra el subjetivismo, Torretti recuerda que el aprendizaje bayesiano presupone (en la versión canónica de De Finetti) sucesos perfectamente intercambiables, como si el mundo estuviera hecho "de eventos bien deslindados en sí mismos de una vez por todas, cuya adecuada clasificación viene dada con ellos" (Torretti, 2003: 19). Para Torretti, no existe tal división platónica de la realidad, y son nuestros intereses y proyectos los que categorizan el mundo. Y sin un acuerdo previo sobre tales categorías, el teorema de Bayes no servirá para ponernos de acuerdo a medida que acumulemos evidencia.

Torretti se siente más cercano de la interpretación propensionista, donde la probabilidad representaría "las propensiones de un arreglo experimental específico" (Torretti, 2003: 28). Por ejemplo, las simetrías físicas de un dispositivo (el equilibrio de una moneda o un dado) nos permiten postular la equiprobabilidad de los sucesos a los que potencialmente puede dar lugar. Para Torretti, la principal resistencia filosófica al propensionismo es "la negativa positivista a reconocer la posibilidad física como una noción primitiva, tan comprensible para todos como la noción de existencia actual" (Torretti, 2003: 32). Y concluye sorprendido de que "tantas personas que la sociedad emplea como pensadores profesionales se nieguen a entender un concepto que un niño de tres años maneja con soltura" (Torretti, 2003: 33). 
En esta versión pragmatista del propensionismo que sugiere Torretti, la probabilidad es indisociable del agente que la usa ${ }^{2}$. El cálculo debe servir a sus decisiones singulares, el espacio de sucesos debe interpretarse según sus intereses y son las posibilidades que discierne en un arreglo experimental las que dan sentido al propio concepto de probabilidad. Históricamente, el enfoque de Torretti nos permite, en efecto, dar cuenta de sus orígenes: fueron los intereses de un jugador, el caballero de Méré, lo que motivó a Pascal para analizar combinatoriamente las posibilidades de un dispositivo aleatorio y formular el concepto que hoy denominamos esperanza matemática (Coumet, 2002). Pero los historiadores de la probabilidad han señalado, sin embargo, cómo los intereses de Pascal (y luego Christiaan Huygens) se articulaban sobre bases normativas: la esperanza de una apuesta señala el justo precio del contrato aleatorio que establecen los jugadores. Aunque Torretti se lo concede, para él la justicia del juego es un aspecto secundario: "ello no impide", afirma, "que los asertos de equiprobabilidad se hayan basado en la percepción de simetrías físicas, donde estas existían" (Torretti, 2003: 6n).

Pues bien, el diálogo que pretendo establecer aquí con Torretti quiere ampliar su propensionismo y mostrar que la normatividad original de la esperanza matemática desempeñó un papel principal en el nacimiento de nuestro concepto moderno de probabilidad. En la primera parte del trabajo, quiero mostrar en qué sentido la esperanza matemática formalizaba un principio de justicia. En la segunda parte ilustraré, apoyándome en los trabajos de G. Shafer y V. Vovk (Shafer \& Vovk, 2001), cómo iluminar sistemáticamente esa normatividad a partir de una concepción de la probabilidad articulada sobre la teoría de juegos. Quizá esto confunda todavía más un concepto como el de probabilidad, ya de por sí sobreinterpretado, según señala el mismo Torretti (Torretti, 2003). Pero espero que sirva también para poner de manifiesto su dimensión pragmática en los términos que el propio Torretti señala: de qué modo pretendía ser una guía para la vida, explotando las posibilidades que los agentes perciben no ya sólo en los objetos, sino en su propia interacción.

El principio sobre el que pivota nuestro argumento es el que denominaremos igualdad en el riesgo: el precio de una apuesta debe ser proporcional al riesgo asumido. Veremos, en primer lugar (sección 2) cómo la fuerza normativa de este principio tiene un origen aristotélico. Para Aristóteles, la justicia de una transacción se basa en la igualdad en el intercambio: tanto aportas, tan-

\footnotetext{
2 Torretti no está sólo en este enfoque pragmatista de la probabilidad. En la Escuela de Stanford, Ian Hacking ya había destacado el papel de los dispositivos aleatorios (chance set-ups; o arreglos como traduce Torretti) como punto de partida de nuestra concepción moderna de la probabilidad (Hacking, 2006). Más recientemente (Suárez, 2011) ha reivindicado una lectura peirceana del propensionismo.
} 
to recibes. No cuestionaremos semejante intuición, sino que veremos cómo se transmite intacta en la tradición escolástica y se modula, incorporando el riesgo, en la discusión de los contratos aleatorios. Veremos después (sección 3) cómo la teoría de la probabilidad comienza con la formalización de esta intuición normativa en el concepto de esperanza matemática. La justicia en el intercambio permite así cuantificar el precio de una apuesta (su valor esperado) aun sin una concepción separada de la probabilidad matemática. Pero si la justicia de un precio depende de su proporción con el riesgo y no tenemos probabilidades para cuantificar tal riesgo, ¿en qué habrá de basarse la esperanza? Veremos (sección 4) cómo los tratadistas del XVII apelaron a la incertidumbre de las mismas partes contratantes: si ambas estiman sus respectivos riesgos como equivalente, sin que ninguna de ellas explote la ignorancia de la otra, el precio que acuerden será justo.

Además de frecuencias, propensiones y creencias, en el origen de la probabilidad encontramos una interacción estratégica en el sentido de la teoría de juegos: dos apostadores han de llegar a un acuerdo sobre el precio de su apuesta. Esta interacción es insignificante desde el punto de vista de la teoría de la medida, sobre la que se funda matemáticamente nuestra concepción contemporánea de la probabilidad. Pero recientemente Glenn Shafer y Vladimir Vovk han propuesto una formulación alternativa que dar prioridad a los juegos: el valor esperado será justamente el precio de una apuesta que un jugador realiza sobre lo que sucederá en el mundo. A partir de este concepto de valor esperado veremos someramente (sección 5) cómo Shafer y Vovk pretenden llegar al concepto de probabilidad.

Desde una perspectiva pragmatista, es interesante advertir cómo la propuesta de Shafer y Vovk explicita los intereses estratégicos de los jugadores al lidiar con el riesgo de una apuesta, de un modo que ilumina la intuición normativa de la que partíamos. Según Shafer y Vovk, el precio exacto de una apuesta será aquel en que coincida el menor precio al que un jugador pueda comprarla y el mayor precio al que pueda venderla. Este sería un precio justo conforme al principio aristotélico de justicia en el intercambio: tanto puso un jugador en la compra de una apuesta, eso mismo recibirá en su venta. En la conclusión (sección 6) veremos cómo la concepción de la probabilidad que nos proponen Shafer y Vovk cumple buena parte de los desiderata de Torretti, pero nos permite también ofrecer iluminar el nacimiento de la probabilidad desde una perspectiva pragmatista que va más allá de los arreglos experimentales. 


\section{Igualdad en el intercambio e igualdad en el riesgo}

Lo que denominaremos igualdad en el intercambio es simplemente el principio de la justicia conmutativa que Aristóteles presentó en el quinto libro de la Ética nicomaquea (EN 1131b25-1132b20) (Aristóteles, Pallí Bonet, \& Lledó, 1985). Aristóteles se plantea aquí el problema de la justicia de los contratos a través de una analogía matemática. Supongamos que las dos partes contratantes tiene iguales derechos sobre un cierto bien, pero han recibido una división desigual $(a, b$, donde $a \neq b)$. Para Aristóteles, la división justa del bien entre las dos partes contratantes es la media aritmética de la división original: $(a+b) / 2$. Aunque Aristóteles no detalla cómo se aplicar esta analogía en un intercambio real, su intuición tuvo una enorme influencia en nuestra tradición occidental y se transmite de modo prácticamente literal a sus comentaristas medievales. Por ejemplo, para el Aquinate, un intercambio justo es aquel en el que las cantidades que cambian de manos no se desvían de la media aritmética del total intercambiado:

Pues si al principio ambas partes tenían 5, y una de ellas recibe 1 de lo que es propio de la otra, una, es decir, la que recibe, tendrá 6 , y a la otra le quedarán 4. Habrá, pues, justicia si se reduce a ambas al término medio, de modo que se quite 1 a la que tiene 6 y que se dé a la que tiene 4; pues de ese modo tendrá cada uno 5, que es el medio. (ST II-II, q61, a2) (Aquino, 1985: 513)

Sobre la base de este principio aristotélico, los escolásticos discutieron ampliamente sobre la justicia de los denominados contratos aleatorios, en los que beneficios y pérdidas dependen de un acontecimiento incierto (Ceccarelli, 2001). La igualdad en el intercambio se modula aquí en igualdad en el riesgo, principio del que presentaremos la versión de Domingo de Soto, uno de los más influyentes teólogos-juristas de la Escuela de Salamanca. Soto sistematizó siglos de controversias jurídicas en su monumental tratado De Iustitia et Iure (1556), en cuyo libro VI, qq6-7 (Soto et al., 1967: 575-581) se discuten los contratos aleatorios. La cuestión que aborda allí Soto es cómo distribuir justamente los beneficios y pérdidas en una sociedad formada mediante tal contrato. Para Soto, una división aristotélica (una media aritmética) solo sería justa si los socios asumiesen riesgos iguales sobre su contribución (sea esta capital o trabajo) a la sociedad. Si los riesgos asumidos son diferentes, la división de beneficios y pérdidas debe ser proporcional a tales riesgos. La igualdad en el riesgo propuesta por Soto incorpora, por tanto, la incertidumbre a la igualdad aristotélica en el intercambio. 
La igualdad en el riesgo le permite distinguir a Soto entre seguros y préstamos. En un préstamo el propietario del dinero no asume ningún riesgo al prestarlo: el prestatario debía devolverlo, con independencia de su éxito o fracaso, más los intereses. De ahí la sombra de usura sobre los préstamos. Sin embargo, en los contratos de seguros se aplica la igualdad en el riesgo, pues ambas partes corren con los suyos. Si no se produce ninguna adversidad, el asegurado perderá la prima de su seguro. Si sucede, el asegurador deberá compensar el capital asegurado. A diferencia del interés en un préstamo, la prima es, por tanto, una compensación por cubrir el riesgo. Ahora bien, podemos preguntarnos qué prima es justo pagar en un contrato de seguros, a lo cual Soto responde que no hay una valoración universal del riesgo. Las partes contratantes deben acordarlo entre ellas:

Hay quienes juzguen a quien con la esperanza de ganar ciento, o mil, se haga cargo del peligro de la nave de un comerciante que tal vez valga veinte o treinta mil. A éstos respondemos que nosotros no discutimos sobre el precio; puede, efectivamente, ser justo o injusto. Esto que lo vean los contratantes. (Soto et al., 1967: 580)

En el siglo XVI, la naciente teoría de la probabilidad formaliza el principio de igualdad en el riesgo, cuando Pascal y Huygens formulan el concepto de esperanza matemática para analizar los problemas de distribución que aparecen en un tipo de contratos aleatorios: las apuestas (Sylla, 2003), (Teira, 2006). En su Tratado sobre el triángulo aritmético (1665), Pascal discute el denominado Problema de los repartos: si un juego de azar se interrumpe, ¿cómo distribuir justamente lo apostado? Para Pascal, "la incertidumbre de ganar es proporcional a la certeza de lo que arriesgamos según la proporción de los riesgos (hasard) de ganancia y pérdida"3. La cuestión es, por tanto, cómo cuantificar tal expectativa. En De ratiociniis in ludo aleae (1657), Huygens propuso el siguiente algoritmo. Supongamos un juego de azar en el que dos jugadores pueden o bien ganar la cantidad $a$ o perder la cantidad $b$ :

Si espero $a$ ó $b$, y puedo obtener con igual facilidad cualquiera de las dos, se dirá que mi esperanza vale $(\mathrm{a}+\mathrm{b}) / 2$. (de Mora Charles, 1989)

Tenemos aquí una media aritmética, conforme a la justicia conmutativa aristotélica, pero también una cuantificación implícita del riesgo asumido por cada jugador. Desde el punto de vista de la probabilidad contemporánea, Hu-

${ }^{3}$ Vid. la carta de Pascal a Fermat del 29 de julio de 1654 (Pascal, Aranguren, \& Dampierre, 1981, pp. 658-666). 
ygens habría formulado el concepto de esperanza matemática, una media de ganan cias y pérdidas $(a, b)$ ponderada por su probabilidad (1/2 para ambas opciones). Sin embargo, Huygens carece todavía de un concepto separado de probabilidad (una entidad matemática): como veremos en la siguiente sección su estimación depende del acuerdo que alcanzan ambos jugadores sobre sus apuestas.

Con la formalización del principio de igualdad en el riesgo, Pascal y Huygens resolvieron el problema de los repartos: si un juego se interrumpe, cada jugador deberá recibir el valor de su esperanza en este punto. Y este será también el principio que fije el justo precio de la apuesta: quienes asumen los mismos riesgos deben pagar un mismo precio.

\section{La equivalencia entre contratos aleatorios}

Huygens no cuantificó los riesgos directamente sirviéndose de probabilidad. Se sirvió de un procedimiento frecuente en la aritmética comercial de la época, estudiando contratos de apuestas y buscando equivalencias entre ellos. En la medida en que dos contratos entrañan los mismos riesgos, su valor esperado debía ser el mismo. A través de estas equivalencias, surge implícitamente una cuantificación de la probabilidad.

He aquí el argumento con el que Huygens formula el concepto de esperanza matemática. Imaginemos un juego en el que ambos jugadores tienen las mismas oportunidades de obtener $a$ o $b$ (donde $a<b$ ). El juego se interrumpe y dos jugadores más se incorporan, cada uno de los cuales paga la cantidad $x$ para jugar (el Problema de los repartos es decidir el valor de $x$ ). Ambos acuerdan que el ganador de este Segundo juego ganará $2 x$ y el perdedor recibirá $a$, como en el primero. El primer y el segundo juego serán equivalentes si el ganador recibe en ambos casos la cantidad pactada en el primero: $b$. Por lo tanto, $2 x$ - $a$ debe ser igual a $b$. Así, la cantidad $\mathrm{x}$ que los dos nuevos jugadores deben pagar para incorporarse al juego debe ser: $(a+b) / 2$. En nuestros términos, este es el valor esperado del juego y, puesto que son equivalentes, será también el justo precio de la apuesta requerida para entrar en el primer juego (interrumpido). Así, la ponderación $1 / 2$ que encontramos en la fórmula de Huygens se basa en el número de jugadores participantes (dos), suponiendo que tienen igual facilidad para ganar y perder. Como señala (Torretti, 2003: 6n), puede argumentarse que el acuerdo se basa en la simetría del dispositivo aleatorio del que se sirven para su apuesta. Pero el razonamiento que permite su acuerdo se basa, advirtámoslo en la percepción común entre las partes contratantes de la justicia del precio así calculado. 
Hasta la formulación canónica de Jacob Bernoulli, los primeros teóricos de la probabilidad se sirvieron durante algunas décadas de esta aproximación contractualista. Así planteó Jan De Witt (1625-1672) en 1671 cómo calcular el precio justo de un seguro 4 . Imaginemos un jugador (Juan) que tiene las mismas oportunidades de obtener diferentes cantidades (e.g., 2000, 3000, 4000 florines). ¿Cómo ha de calcular el valor de sus expectativas? Para ello, argumenta De Witt, Juan tiene que firmar un contrato con otros dos jugadores, Pedro y Pablo. Cada uno de ellos contribuirá con 3000 florines a un fondo conjunto. Estos 9000 florines los recibirá uno de los tres en una lotería en la que cada uno de ellos tiene las mismas oportunidades de ganar. Juan tendrá que firmar además dos contratos separados con Pedro y Pablo: si Juan o Pedro gana la lotería, compensará al otro con 2000 florines; si Juan o Pablo ganan la lotería, el perdedor será compensado con 3000 florines. Estos tres contratos le permiten a Juan tener las mismas oportunidades de ganar o bien 4000 florines (el fondo conjunto, menos la compensación a sus socios), o bien 2000 florines o 3000 florines (si gana Pedro o Pablo). Pedro puede esperar ganar 3000 florines, que es el precio justo de la apuesta ${ }^{5}$.

Para De Witt, este ejemplo ilustra un principio general: el valor de una expectativa es igual a la suma de las cantidades en juego dividido por las oportunidades de obtenerlas -9000/3 en el ejemplo. Como Huygens, De Witt utiliza el número de jugadores para expresar la equiprobabilidad. Cuando no se supone igualdad de oportunidades entre los jugadores, su algoritmo necesita tantos jugadores en el contrato inicial como la suma de las oportunidades de obtener los distintos resultados: por ejemplo, si las oportunidades son 6,4 y 3 , debe haber 13 jugadores.

Por lo tanto, las probabilidades no existen separadas de los contratos en los que se formalizan. Para Huygens y De Witt, la expectativa es el concepto primitivo. Por lo tanto, una vez firmados los contratos, podríamos juzgar que, en la medida en que son justos y su precio es el mismo, los riesgos son los mismos para todas las partes. Podemos preguntarnos, sin embargo, cómo evalúan sus riesgos los jugadores en cada apuesta, antes de firmar el contrato.

\footnotetext{
4 "I presuppose that the real value of certain expectations or chances of objects, of different value, must be estimated by that which we can obtain from several expectations or chances, dependent on one or several equal contracts." Citamos a partir de la traducción inglesa de Hendricks (Barnwell \& Hendricks, 1856: $82-83)$

${ }^{5}$ Aunque De Witt no lo menciona, para garantizar la igualdad de oportunidades de todos los jugadores sería necesario añadir al esquema anterior un contrato adicional entre Pedro y Pablo, por el cual, si alguno de los dos gana el premio de 9000 florines, el ganador entregará al perdedor la suma de 3000 florines. De esta forma los tres jugadores tendrían exactamente las mismas expectativas de ganancias en el juego.
} 


\section{La justicia de un contrato aleatorio}

En efecto, para poder aplicar el principio de igualdad en el riesgo en el análisis de contratos equivalentes debemos suponer que las partes contratantes han estimado sus riesgos iguales. ¿Y cómo podrían haberlo hecho sin una definición previa de probabilidad? De nuevo, el Derecho nos proporciona una posible respuesta. Pascal contaba en su círculo con el jurista Jean Domat (1625-1696), autor de otro monumental tratado Las leyes civiles en su orden natural (1689), considerado hoy como una de las primeras sistematizaciones racionalista del derecho francés.

Domat discute en varios puntos el papel de la incertidumbre en la justicia de un acuerdo. Por ejemplo, aquellos pactos sobre un suceso incierto en los que una de las partes puede, si se produce, renunciar a cualquier beneficio y exonerarse de cualquier pérdida (Libro I, Título I, Sección IV, XX). Para Domat, la justicia de semejante pacto se fundamenta en lo siguiente:

Esta forma de pactos tienen su justicia en que una [de las partes] prefiere como reparto algo cierto y conocido, sea en beneficios o pérdidas, en vez de la incierta espera de los acontecimientos. La otra, por el contrario, encuentra ventajoso en el reparto esperar una condición mejor. Así se produce entre ambas [partes] una especie de igualdad en el reparto, que hace justo su pacto. (Domat, 1689: 97)

Si las partes contratantes tienen expectativas complementarias sobre el resultado, el acuerdo es justo. Sus expectativas dependen, por supuesto, de una estimación subjetiva del riesgo de que se produzca un suceso incierto. Para Domat esta estimación subjetiva basta para fundar la justicia de su acuerdo $s i$ ambas partes están en igualdad de condiciones respecto al azar. Por ejemplo (Libro I, Título VIII, Sección II, XI), en una sociedad universal se acuerda que la dote de las hijas de los socios se tomará del fondo común. Si resulta que solo uno de estos tiene hijas, será justo que disponga de la dote, puesto que en el momento en el que se crea la sociedad "todos estaban en la misma incertidumbre ante el acontecimiento [tener una hija], y con igual derecho. Siendo su condición igual, será justo su pacto" (Domat, 1689: 308).

Por lo tanto, no se trata de que las partes contratantes sean capaces de cuantificar sus riesgos. Más bien, deben asegurarse de que la incertidumbre es la misma para ambas partes: ninguno de los socios debe saber más que los demás acerca de sus respectivas probabilidades de tener hijas. Ninguno de ellos pudo, por tanto, explotar la ignorancia ajena. Para que un contrato sea válido, afirma Domat, basta con que las partes sean conscientes de las obliga- 
ciones que contraen y acepten su igualdad ante el riesgo. Por ejemplo (Libro I, Título XVIII, preámbulo), si al negociar el reparto de una herencia, todos los herederos ignoran por igual la existencia de deudas o cargas, su aparición posterior no les justifica a impugnar el reparto.

Pues no es sobre un conocimiento exacto y entero del detalle de los derechos y cargas de sucesión que se funda su compromiso. Basta para fundarlo y convertirlo en irrevocable que se sepa que una herencia consiste en derechos y cargas, que a menudo los herederos, incluso los más clarividentes, desconocen. [Basta que el heredero] sepa que en la mayor o menor incertidumbre de lo que no podemos conocer, él ha optado por un reparto con un riesgo de pérdida o beneficio en un bien de naturaleza incierta. (Domat, 1689: 489)

Por lo tanto, es la igualdad de condiciones ante la aleatoriedad de un contrato lo que determina su validez, aun sin una cuantificación precisa de sus riesgos. Podemos plantearnos ahora si es posible sobre esta base contractualista acceder a una definición matemática de la probabilidad.

\section{Probabilidades y precios}

Hemos visto cómo la esperanza matemática define el justo precio de una apuesta si aceptamos el principio aristotélico de igualdad en el riesgo. Esta igualdad se define, además, sobre una estimación contractual del riesgo, sin una cuantificación explícita de la probabilidad. Desde el punto de vista de nuestra definición axiomática de probabilidad, la interacción entre jugadores no tiene, desde luego, significación. Pero recientemente Glenn Shafer y Vladimir Vovk han propuesto una concepción de la probabilidad en la que esta se define a partir de la interacción estratégica entre dos jugadores que deciden sobre el precio de una apuesta. Siguiendo su propia presentación introductoria (Shafer \& Vovk, 2001: cap. 1), esbozaremos aquí los rudimentos de esta concepción para discutirla en la sección siguiente.

Supongamos entonces un juego con dos jugadores que denominaremos Escéptico y Mundo. Escéptico apuesta sobre qué ocurrirá; Mundo decide qué ocurrirá. Es un juego de información perfecta y completa para ambos. Podemos imaginar que Escéptico tiene una teoría sobre lo que ocurrirá en el mundo y apuesta sobre tales sucesos de acuerdo con su teoría. Según Shafer y Vovk, necesitamos dos principios para dar sentido probabilístico a este juego.

Por un lado, las apuestas de Escéptico se regirán por el principio de cobertura dinámica (Dynamic Hedging). Tenemos apuesta simples (sobre un solo 
movimiento de Mundo) y apuestas complejas (sobre varios movimientos de Mundo): cuando las apuestas complejas son combinaciones de apuestas simples, el precio de estas últimas determina el de las primeras. En cada ronda del juego, Escéptico puede realizar apuestas simples o complejas.

Por otro lado, Shafer y Vovk nos proponen la que denominan su hipótesis interpretativa fundamental. La hipótesis afirma que es raro que ocurran sucesos con una probabilidad baja o nula. Adoptar la hipótesis nos permite poner a prueba la teoría de Escéptico a través de las apuestas: si ocurren sucesos con una probabilidad baja o nula, la teoría no nos proporcionará un buen modelo del mundo ${ }^{6}$.

Un juego probabilístico es una especificación completa de los movimientos disponibles para los jugadores. Para definir estos movimientos necesitamos (i) un espacio muestral de sucesos posibles (las secuencias de movimientos de Mundo) y (ii) apuestas que definimos como variables cuyos valores son precios. Podemos representar el espacio muestral como un árbol en el que de cada nodo (o situación) parten los posibles movimientos de Mundo. Cuando un recorrido a través de distintos nodos termina, la situación terminal (las ganancias o pérdidas) identifica el recorrido completo.

Cada movimiento de Escéptico es una apuesta, definida por su precio y su premio. Este dependerá del siguiente movimiento de Mundo. Se pueden realizar apuestas por cualquier fracción o múltiplo de las apuestas disponibles. Escéptico puede además endeudarse sin intereses para apostar. Un protocolo de apuestas es simétrico si Escéptico puede comprar una apuesta con premio $x$ al precio $m$ y vender esa misma apuesta por $-m$.

Escéptico puede formar estrategias $P$ : planes para apostar en cada nodo (no terminal) del espacio muestral. El capital de Escéptico viene determinado por su capital inicial y su estrategia. Dada una estrategia $P$ y una situación $t$, $K^{P}(t)$ será el capital de Escéptico en $t$, si inicia el juego con capital 0 y sigue la estrategia $P$. Supuesto que $\xi$ sea un recorrido completo, $K^{P}(\xi)$ será el capital final del Escéptico al concluir $\xi$.

Sea $x$ una variable representando una apuesta con premio $x$ que Escéptico puede comprar o vender al precio $\alpha$. La transacción será satisfactoria si se cumple la condición siguiente:

\footnotetext{
${ }^{6}$ A diferencia de otras formulaciones, Shafer y Vovk insisten en que su hipótesis no se basa en las probabilidades de los sucesos particulares sobre los que se formulan las apuestas. Es su propia versión del denominado puente de Cournot para conectar la teoría de la probabilidad con el mundo (Shafer \& Vovk, 2001, p. 56).
} 


$$
\text { Para cualquier } \xi, K^{P}(\xi) \geq x(\xi)-\alpha
$$

Es decir, el capital acumulado por Escéptico en el nodo final del recorrido $\xi$, siguiendo la estrategia $P$ es mayor o igual que el premio $x(\xi)$ recibido al final de $\xi$ menos el precio que pagó por la apuesta.

Shafer y Vovk definen el precio superior de $x$ (su coste) de la siguiente manera:

$$
\bar{E}(x) \equiv \inf \left\{\alpha \mid \text { hay una estrategia } P \text { tal que } K^{p} \geq x-\alpha\right]
$$

$\bar{E}(x)$ es el menor precio al que Escéptico puede comprar la apuesta $x$ en el espacio muestral. Correlativamente, el mayor precio al que Escéptico puede vender $x$ (su valor residual) se define como sigue:

$$
\underline{E}(x) \equiv \sup \left\{\alpha \mid \text { hay una estrategia } P \text { tal que } K^{p} \geq \alpha-x\right]
$$

Vender $x$ por $\alpha$ es equivalente a comprar $-x$ por $-\alpha$. Es decir, para todo $x$ se verifica:

$$
\bar{E}(x)=-\underline{E}(-x)
$$

En una situación t, un protocolo de apuestas es coherente si se cumple, para todo $x$ :

$$
\underline{E_{t}} x \leq \overline{E_{t}} x
$$

En un protocolo de apuestas coherente, no es seguro que Escéptico pueda ganar dinero: el menor precio al que Escéptico puede comprar una apuesta es menor o igual que el mayor precio al que puede venderla.

El precio (exacto) de $x$ en $t$ será $E_{t} x$ cuando se cumpla:

$$
\underline{E_{t} x}=\overline{E_{t}} x
$$

Los precios así definidos tienen las propiedades de la esperanza matemática en teoría de la probabilidad, aun cuando Shafer y Vovk no han definido todavía probabilidad alguna.

La hipótesis interpretativa fundamental nos permite ahora dar sentido a los precios en un juego probabilísticos. La hipótesis afirma, en su versión para casos finitos, que no hay ninguna estrategia que le permita a Escéptico simultáneamente (1) estar seguro de que evitará la bancarrota y (2) tener una oportunidad razonable de enriquecerse -es decir, multiplicar significativa- 
mente su capital inicial. Un suceso será tanto más improbable cuanto mayor sea el enriquecimiento que permita. En un protocolo coherente, Mundo puede siempre impedir que Escéptico se enriquezca, cumpliendo con la hipótesis.

Shafer y Vovk definen entonces el concepto de probabilidad a partir de los precios superior e inferior. En primer lugar, definen la variable $\mathrm{I}_{E}$ de la siguiente manera: dado un suceso $K$ y un recorrido $\xi$

$$
I_{K}(\xi):= \begin{cases}1 & \text { si } \xi \in K \\ 0 & \text { si } \xi \notin K\end{cases}
$$

Así la probabilidad superior se define como: $\bar{P} K:=\bar{E} I_{E}$ La probabilidad inferior será así: $\underline{P} K:=\underline{E} I_{E}$

Si el protocolo es coherente, la probabilidad superior e inferior verifican ${ }^{7}$ :

$$
\begin{gathered}
0 \leq \underline{P} K \leq \bar{P} K \leq 1 \\
\underline{P} K=1-\bar{P} K^{\mathrm{C}}
\end{gathered}
$$

La hipótesis fundamental nos permite interpretar estas probabilidades en términos de precios: si, por ejemplo, la probabilidad superior de $K$ es muy baja, Escéptico puede comprar la variable indicadora de $K, I_{K}$, por un precio muy bajo. Es seguro que evitará la bancarrota, pues el precio de $I_{K}$ es mayor que cero. Y se enriquecerá si $K$ sucede, cosa improbable.

\section{Un pragmatismo ampliado}

Aceptemos, en aras del argumento, que la concepción de la probabilidad que nos proponen Shafer y Vovk es viable, y es posible obtener a partir de sus principios las leyes de los grandes números y el teorema central del límite. Examinemos brevemente de qué modo enfrentaría las objeciones de Torretti y en qué sentido permitirían expandir su pragmatismo.

Contra el frecuentismo, recordémoslo, Torretti señala que no nos permite interpretar la probabilidad como guía de la vida, al no permitirnos asignársela a un suceso singular. Shafer y Vovk no parten, sin embargo, de una medida de probabilidad (en el sentido de la teoría de la medida) en la que hayamos de interpretar tales sucesos singulares. Escéptico asigna probabilidades a cada suceso sobre el que apuesta sirviéndose de la información de la que dispone,

\footnotetext{
${ }^{7} K^{c}$ es el suceso complementario de $K$ en el espacio muestral, los movimientos de Mundo que no están en $K$ o donde $K$ no sucede.
} 
de acuerdo con las dos hipótesis de partida. Mundo decide. El juego tendrá, por tanto, $n$ probabilidades para los sucesos sobre los que se apuesta, $p_{1}, \ldots$, $p_{n}$, no una medida de probabilidad completa (Shafer \& Vovk, 2001: 58).

Sin embargo, los precios teorizados por Shafer y Vovk no son siempre directamente interpretables en términos probabilísticos. Si la probabilidad superior y la probabilidad inferior están ambas próximas a 0 o a 1 , podremos decir que el suceso es muy probable o improbable. Pero si la probabilidad inferior y la superior no están alineadas cerca de 0 y 1 , no tendremos evidencia suficiente para asignarle probabilidad directamente al suceso (Shafer \& Vovk, 2001: 16). Por lo tanto, a diferencia del Bayesianismo, no necesita tampoco suponer que el mundo se componga de "eventos bien delimitados" con una probabilidad precisa (y su correspondiente precio).

El precio a pagar, sin embargo, es que si sólo las probabilidades cercanas a 1 y a 0 tienen sentido independiente, cualquier otro valor intermedio sólo tendrá sentido a través de la repetición del suceso, al modo frecuentista, tal como sugiere la hipótesis fundamental. Shafer y Vovk recurren para ello a las leyes de convergencia que prueban a partir de teoría de juegos (Shafer \& Vovk, 2001: 44).

Por lo tanto, esta versión de la probabilidad no será en todos los casos una guía para la vida, en el sentido deseado por Torretti. Esto es, una propensión definida sobre un arreglo experimental singular, y no sobre la secuencia de resultados que el arreglo genera (Suárez, 2011). Como indican (Shafer \& Vovk, 2001: 46), el suyo es un enfoque fundamentalmente propensionista ${ }^{8}$ : la teoría de juegos considera los cursos alternativos de acción que se pueden producir en una apuesta, según decidan Escéptico y Mundo. Las probabilidades mediría aquí, por tanto, posibilidades (potencialities) en el juego.

Sin embargo, el enfoque de Shafer y Vovk es menos exigente que las versiones habituales del propensionismo: no es necesario partir de arreglos experimentales con simetrías bien definidas. Basta con que el fenómeno modelizado manifieste regularidades parciales, sobre las que se puedan asignar precios, pues tampoco es necesario aquí partir de una medida de probabilidad completa.

\footnotetext{
${ }^{8}$ Sin embargo, se puede acomodar tanto el frecuentismo como el bayesianismo en el enfoque de Shafer y Vovk (Shafer \& Vovk, 2001: 19-20). Los precios se pueden basar en frecuencias observadas en el pasado o en las elecciones personales entre riesgos (articuladas a partir de apuestas coherentes). En este último caso, se prescinde de la hipótesis fundamental, pues las probabilidades se refieren a riesgos asumidos, no a creencias subjetivas sobre lo que ocurrirá.
} 
Por tanto, en un plano sistemático, la propuesta de Shafer y Vovk capta buena parte de las intuiciones de Torretti. Pero, desde nuestro punto de vista, nos permite ampliarlas decisivamente en el plano histórico. Pues el nacimiento de la teoría de la probabilidad en la matematización de los juegos de azar dejaría de ser una casualidad -en el sentido que reivindicó (Coumet, 2002). Desde la perspectiva de Shafer y Vovk, es posible llegar desde los precios a la esperanza matemática, y de aquí a una definición de probabilidad.

Cuando el menor precio al que Escéptico puede comprar una apuesta coincide con el mayor precio al que Escéptico puede venderla (sección 5), tenemos su precio exacto, con las propiedades de la esperanza:

$$
\underline{E_{t}} x=\bar{E}_{t} x
$$

Esta igualdad entre precios sobre la que pivota esta concepción de la probabilidad es justamente la intuida a través del principio aristotélico de igualdad en el intercambio, como veíamos en la sección 2: no hay ganancia en la compraventa, cada cual aporta exactamente lo que recibe. El principio de cobertura dinámica se encuentra ya implícito en las equivalencias entre contratos aleatorios, tal como presentamos en las secciones 2 y 3 (Shafer \& Vovk, 2001: 31). La intuición financiera que subyace a este principio es que si un jugador dispone del capital A necesario para obtener, con una combinación de apuestas, una cantidad B, diremos que A es el precio de B. Los riesgos de los jugadores se cuantifican a través de los precios de los contratos, sin una concepción separada de la probabilidad: mismo precio, mismos riesgos. Los jugadores contratantes sólo necesitan coincidir en una estimación concordante del riesgo, tal como advertía Domat (sección 4). Esta estimación es pre-teórica: basta con que ninguna de las partes pueda explotar la ignorancia de la otra sobre las incertidumbres en las que basan su contrato aleatorio. La aleatoriedad debe ser, a priori, real, no ha de dar lugar a una martingala.

Obviamente todas estas son interpretaciones retrospectivas que no gustarán al historiador de la probabilidad más escrupuloso. Pero sigo aquí la inspiración del mismo Torretti, en un simposio que organicé en 2010 sobre algunos de sus libros. Decía allí:

Si hay todavía historiadores que se crean capaces de describir el pasado wie es eigentlich gewesen, yo sería el último en negarles su derecho a intentarlo. Siento un gran respeto y gratitud hacia lo que Nietzsche llamó la historia anticuaria, tengo en menos pero también le debo mucho a lo que llamó historia 
monumental, pero yo mismo no me he propuesto nunca practicar otro género de historia que la que él llamó crítica (Vom Nutzen und Nachteil der Historie für das Leben, en Unzeitgemässe Betrachtungen). (Torretti, 2010: 148)

La propuesta de Shafer y Vovk nos permite, en mi opinión, hacer una historia crítica de un episodio central en el nacimiento del concepto moderno de probabilidad. Me hubiera gustado poder discutirlo aquella tarde en Santiago de Chile con Torretti, pero quede aquí al menos como testimonio de mi gratitud y admiración.

\section{Referencias}

Aquino, T. d. (1985). Suma de Teología III. Parte II-II (a). Madrid: BAC.

Aristóteles, Pallí Bonet, J., \& Lledó, E. (1985). Etica nicomáquea ; Etica eudemia. Madrid ,: Gredos.

Barnwell, R. G., \& Hendricks, F. (1856). A sketch of the life and times of John De Witt, grand pensionary of Holland, to which is added, his treatise on life annuities. New York,: Pudney \& Russell.

Ceccarelli, G. (2001). «Risky business: Theological and canonical thought on insurance from the thirteenth to the seventeenth century». Journal of Medieval and Early Modern Studies, 31(3), 607-658.

Coumet, E. (2002). «La teoría del azar, ¿nació por azar?». Empiria. Revista de metodología de ciencias sociales(3), 33. doi:10.5944/empiria.3.2000.877.

de Mora Charles, M. (1989). Los inicios de la teoría de la probabilidad. Siglos XVI y XVII Bilbao: Universidad del País Vasco.

Domat, J. (1689). Les Loix civiles dans leur ordre naturel París: J. B. Coignard.

HACKING, I. (2006). The emergence of probability : a philosophical study of early ideas about probability, induction and statistical inference (2nd ed.). Cambridge ; New York: Cambridge University Press.

Pascal, B., Aranguren, J. L. L., \& Dampierre, C. R. d. (1981). Obras. Madrid: Alfaguara.

Shafer, G., \& Vovk, V. (2001). Probability and finance : it's only a game! New York: J. Wiley \& Sons. 
Soto, D. d., Carro, V. D., \& Instituto de Estudios Políticos (Madrid). (1967). De iustitia et iure libri decem = De la justicia y del derecho : en diez libros (Edición facsimilar de la hecha por D. de Soto en 1556, con su versión castellana correspondiente ed.). Madrid: Instituto de Estudios Políticos.

SuÁrez, M. (2011). «Propensities and Pragmatism». Journal of Philosophy, 110(2), 61-102.

Sylla, E. D. (2003). «Business ethics, commercial mathematics, and the origins of mathematical probability». History of Political Economy, 35(5), 309-337.

TeIRA, D. (2006). «On the normative dimension of the St. Petersburg paradox». Studies in History and Philosophy of Science Part A, 37(2), 210223.

ToRretti, R. (1998). El paraíso de Cantor. La tradición conjuntista en filosofía de la matemática. Santiago de Chile: Universitaria.

Torretti, R. (2003). «El concepto de probabilidad». Diálogos, 38, 407-448.

Torretti, R. (2010). «Respuestas a mis críticos». Teorema, XXIX(1), 147151. 